\title{
Towards Federated Learning-Enabled Visible Light Communication in 6G Systems
}

\author{
Shimaa Naser, Student Member, IEEE, Lina Bariah, Member, IEEE, Sami Muhaidat, Senior Member, IEEE, \\ Mahmoud Al-Qutayri, Senior Member, IEEE, Ernesto Damiani, Senior Member, IEEE, and Paschalis C. \\ Sofotasios, Senior Member, IEEE
}

\begin{abstract}
Visible light communication is envisaged as a promising enabling technology for sixth generation (6G) and beyond networks. It was introduced as a key enabler for reliable massive-scale connectivity, mainly thanks to its simple and low-cost implementation which require minor variations to the existing indoor lighting systems. The key features of VLC allow offloading data traffic from the current congested radio frequency (RF) spectrum in order to achieve effective short-range, high speed, and green communications. However, several challenges prevent the realization of the full potentials of VLC, namely the limited modulation bandwidth of light emitting diodes, the interference resulted from ambient light, the effects of optical diffuse reflection, the non-linearity of devices, and the random receiver orientation. Meanwhile, centralized machine learning (ML) techniques have exhibited great potentials in handling different challenges in communication systems. Specifically, it has been recently shown that ML algorithms exhibit superior capabilities in handling complicated network tasks, such as channel equalization, estimation and modeling, resources allocation, opportunistic spectrum access control, non-linearity compensation, performance monitoring, detection, decoding/encoding, and network optimization. Nevertheless, concerns relating to privacy and communication overhead when sharing raw data of the involved clients with a server constitute major bottlenecks in large-scale implementation of centralized ML techniques. This has motivated the emergence of a new distributed ML paradigm, namely federated learning (FL). This method can reduce the cost associated with transferring the raw data, and preserve clients privacy by training ML model locally and collaboratively at the clients side. Thus, the integration of FL in VLC networks can provide ubiquitous and reliable implementation of VLC systems. Based on this, for the first time in the open literature, we provide an overview about VLC technology and FL. Then, we introduce
\end{abstract}

This work was supported by Khalifa University under Grant KU/FSU8474000122 and Grant KU/RC1-C2PS-T2/8474000137.

S. Naser, L. Bariah, and E. Damiani are with the Center for Cyber-Physical Systems, Department of Electrical Engineering and Computer Science, Khalifa University, Abu Dhabi 127788, UAE, (e-mails: \{100049402,ernesto.damiani\}@ku.ac.ae, lina.bariah@ieee.org).

S. Muhaidat is with the Center for Cyber-Physical Systems, Department of Electrical Engineering and Computer Science, Khalifa University, Abu Dhabi 127788, UAE, and also with the Department of Systems and Computer Engineering, Carleton University, Ottawa, ON K1S 5B6, Canada, (e-mail: muhaidat@ieee.org).

M. Al-Qutayri is with the Systems-on-Chip (SoC) Center, Department of Electrical Engineering and Computer Science, Khalifa University, Abu Dhabi 127788, UAE, (e-mail: mahmoud.alqutayri@ku.ac.ae).

P. C. Sofotasios is with the Center for Cyber-Physical Systems, Department of Electrical Engineering and Computer Science, Khalifa University, Abu Dhabi 127788, UAE, and also with the Department of Electrical Engineering, Tampere University, Tampere 33014, Finland, (e-mail: p.sofotasios@ieee.org).
FL and its integration in VLC networks and provide an overview on the main design aspects. Finally, we highlight some interesting future research directions of FL that are envisioned to boost the performance of VLC systems.

\section{INTRODUCTION}

$\mathbf{T}$ He recent advancements in indoor lighting systems, accompanied with the revolutionary solid-state progression in light emitting diodes (LEDs), have motivated the emergence of the visible light communication (VLC) concept. In particular, the ability of LEDs to switch rapidly between different light intensity levels enables short range data transmission without affecting the illumination function of LEDs, rendering VLC a cost-effective easy-to-implement technology. VLC systems have enabled a swarm of wireless applications, such as indoor navigation, healthcare, underwater communication, positioning systems, and vehicular communications. The key driver underlying the emergence of such applications are the promising features of VLC systems, such as high data rates, inherent secure communication, enhanced capacity, and ultralow end-to-end latency.

On the other hand, machine learning (ML) is a subfield of artificial intelligence (AI), which has been recently identified as an appealing data-driven solution for optical wireless networks [1]. In centralized ML algorithms, mobile nodes share their data, which are then uploaded, processed, trained, and aggregated at cloud-based servers. Nevertheless, the drawbacks of such cloud-centric algorithms are threefold. Firstly, data privacy is compromised in cloud-based ML because participating devices are requested to send their private data to centralized servers, exposing these data to potential eavesdroppers. Secondly, centralized ML suffers from long propagation delay, rendering it unsuitable for realtime applications. Thirdly, data transmission yields increased network overhead, which renders the implementation of centralized ML in resources-constrained internet-of-things (IoT) devices challenging. This ultimately calls for a fundamental transition from conventional centralized algorithms into novel paradigms, in which the network can be trained in a distributed manner.

In this respect, Federated Learning (FL) was recently considered an efficient tool to train wireless networks, without leaking private information or consuming network resources. 
Specifically, the enhanced on-board computational and storage capabilities of mobile devices with the local datasets are leveraged to enable decentralized local training. It is important to note that adopting FL in VLC systems is particularly appealing in accommodating the ever-growing demand of datadriven privacy-sensitive applications. With this motivation, this article presents an overview of the implementation of FL in VLC systems along with useful theoretical and technical insights.

\section{A. Visible Light Communication}

It is recalled that VLC technology is both particularly effective and rather simple to implement. The main principle of VLC systems is to use LEDs or laser diodes at the transmitter for modulating light intensity, and a photo-detector (PD) at the receiver for converting light intensity into electrical signal for detection purposes. Furthermore, the largely underutilized and license-free visible light spectrum allows VLC to offload data traffic from the current band-limited congested radio frequency (RF) spectrum. Also, VLC systems are inherently secure and are characterized by a high degree of spatial reuse and immunity to electromagnetic interference. Nevertheless, VLC systems are prone to a number of performance-limiting factors, such as ambient light interference, non-linearity of the LEDs, user mobility, and receivers' orientations.

\section{B. Federated Learning}

ML algorithms have been shown particularly effective in handling complicated network tasks, such as channel equalization, estimation and modeling, resources allocation and network optimization. By exploiting the data generated from IoT and smart devices, advanced ML tools use these data for network training purposes [2]. In conventional centralized ML approaches, raw data generated and stored at local devices (clients) are shared with centralized servers or data centers, where are then processed and used in the training process to evaluate a global model. Then, the global model is globally utilized for centralized network optimization. However, despite the promising capabilities of centralized ML techniques, they suffer from privacy issues because participating devices are requested to share their private data with a centralized server. Moreover, the enormous amount of data shared by the participating devices yields an increased communication overhead. Finally, centralized training leads to higher latency and waiting time.

The accelerating advancement in the power, storage, computing, sensing, and communication capabilities of IoT and smart devices has motivated the emergence of a new artificial intelligence sub-field, referred as FL, which aims to overcome privacy risk and communication overhead experienced in traditional centralized learning techniques. FL allows a set of local clients to locally and collaboratively participate in the training process of a global model without having to upload their raw local data to centralized servers. The role

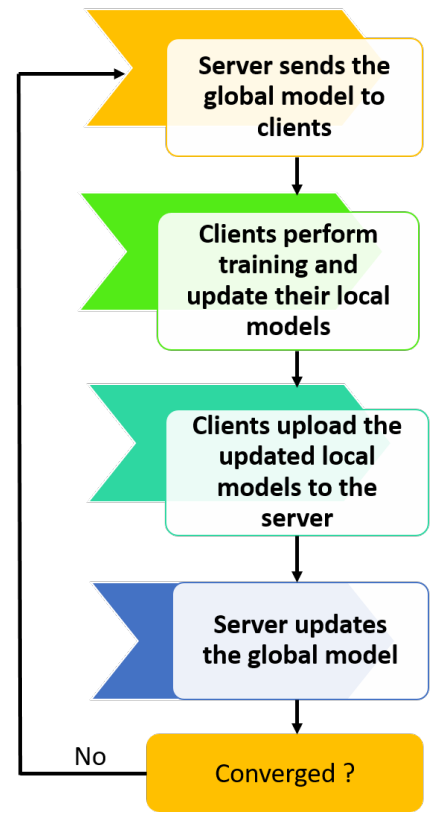

Fig. 1: Learning steps in federated learning.

of the centralized servers in FL is to orchestrate the learning process, coordinate the participating clients, and aggregate the local model parameters received from clients. Specifically, a subset of local clients in FL-based systems are selected and updated with initial model parameters in order to participate in the training process. Subsequently, local training process is preformed at the clients' side using their local datasets. Then, the evaluated local models from all clients are transmitted to a centralized server, where these models are aggregated and trained to obtain the global model. The typical learning steps in FL is summarized in Fig.1.

\section{Related Work}

Inspired by the promising advantages of FL for communication and networking, significant research efforts have been devoted to explore FL in terms of architecture, challenges, design aspects, and applications. Particularly, [3] presented a comprehensive survey that highlights the fundamentals, applications, enabling technologies, and learning mechanisms of FL. On the contrary, [4] discussed open research problems and challenges associated with FL, such as communication efficiency, data privacy, data heterogeneity and model aggregation. From a different perspective, [5] presented the FL taxonomy, with emphasis on the main FL components, including data distribution, ML model, privacy mechanism, communication architecture, scale of federation, and motivation of federation. In [12], FL was explored from a security perspective, while [6] discussed FL in mobile edge computing. In the same context, [7] considered the characteristics, challenges, and future directions of FL in massive-scale networks while [8] addressed the implementation and applications of 
TABLE I: Recent surveys on FL.

\begin{tabular}{|c|l|}
\hline Reference & Main focus of the survey \\
\hline \hline$[3]$ & $\begin{array}{l}\text { Discussion about the challenges of FL and different techniques to address these challenges. } \\
\text { Highlighting on some applications of FL in communication and networking. }\end{array}$ \\
\hline$[4]$ & Extensive discussion about recent advances, challenges, and open problems in FL. \\
\hline$[5]$ & Categorization of FL. \\
\hline$[6]$ & FL in mobile edge networks. \\
\hline$[7]$ & Characteristics, challenges, and future directions for FL in massive networks. \\
\hline$[8]$ & FL and its applications and challenges in 5G networks. \\
\hline$[9]$ & $\begin{array}{l}\text { A comprehensive study of FL and its enabling software and hardware platforms, protocols, real-life } \\
\text { applications and use-cases were discussed. }\end{array}$ \\
\hline$[10]$ & $\begin{array}{l}\text { Threats models and major attacks in FL. } \\
\text { giestigation of federated vehicular networks, their high-level architecture and enabling technolo- } \\
\text { Finally, discussing possible future research direction and open problems. }\end{array}$ \\
\hline
\end{tabular}

FL in the fifth generation (5G) networks. A comprehensive study of FL and its enabling software and hardware platforms, protocols, real-life applications and use-cases was carried-out in [9]. Finally, the key techniques and fundamental assumptions adopted by various attacks in FL were explained in [10]. Then, the authors provided a discussion about future research directions towards more robust privacy preservation in FL. For convenience, the related reported contributions are summarized in Table I.

Notably, the aforementioned contributions considered the implementation of FL in RF scenarios. Also, the interplay between FL and VLC systems is barely addressed in the literature. Accordingly, for the first time, this article provides an overview on the implementation of FL in VLC systems, sheding light on the design aspects of FL in VLC. Furthermore, it constructs a road-map towards open research directions that need considerable investigation.

\section{Federated Learning IN VLC}

\section{A. Fundamentals}

A typical VLC system that employs FL is illustrated in Fig. 2. The appealing features of VLC, such as inherent security, high data rate transmission, energy efficiency and high spatial reuse are the main drivers for utilizing VLC with FL, in order to provide secure, accurate, and fast global model evaluation.

In the context of VLC, multiple LEDs connected through $\mathrm{RF}$ or optical fiber links to a gateway and then to a server represent an interface between the cloud-based server and the participating clients. Specifically, the LEDs are exploited at the downlink communication to assist with global model transmission. Accordingly, the participating clients are determined based on the field of view (FoV) of the LEDs. Therefore, clients existing in the LEDs' coverage area can only take part of the learning process. Also, local model updates are communicated with a gateway, connected to the cloud-based server, through uplink RF or infrared links. This is attributed to the clients limited energy and the undesired radiance from the clients devices. On the other hand, VLC can be leveraged to offload model update traffic in the downlink from the overcrowded RF spectrum to the visible light band, allowing enhanced allocation of the bandwidth resources in the uplink communication.

The centralized cloud-based server in such scenarios is responsible of fulfilling the following tasks: i) initialize the global model evaluation process for a particular learning task; ii) select the participating clients based on different metrics, including the LEDs' coverage area, the clients mobility, clients' receivers orientation, etc; and iii) coordinate the learning process and model aggregation. Hence, $K$ clients, out of a set comprising $N$ nodes, are selected to receive the initial global model parameters $\mathbf{w}^{o}$, aiming to engage them in the learning process. The $k^{t h}$ client will utilize its dataset $\mathfrak{D}_{k}$, which is stored locally, for training. Each dataset is assumed to be composed of $D_{k}$ input-output pair vectors $\left(\mathbf{x}_{k}, \mathbf{y}_{k}\right)$. Assuming stochastic gradient descent at the $i^{t h}$ communication round, the $k^{\text {th }}$ client calculates the gradient of the loss function, namely

$$
F_{k}\left(\mathbf{w}^{i}\right)=\frac{1}{D_{k}} \sum_{j \in \mathfrak{D}_{k}} f_{j k}\left(\mathbf{w}^{i}\right)
$$

where $f_{j k}\left(\mathbf{w}^{i}\right)$ is the loss function of the $j^{t h}$ input-output sample, which could be a linear regression, neural network, or logistic regression.

\section{B. Model Aggregation}

After receiving all local gradients update from participating clients in the $i^{\text {th }}$ communication round, the centralized server performs aggregation in order to compute the global model parameters. An aggregation model, referred to as federated averaging (FedAvg), is used for this in which all local parameters are combined using model averaging. To calculate the new global model parameters, the centralized server performs 


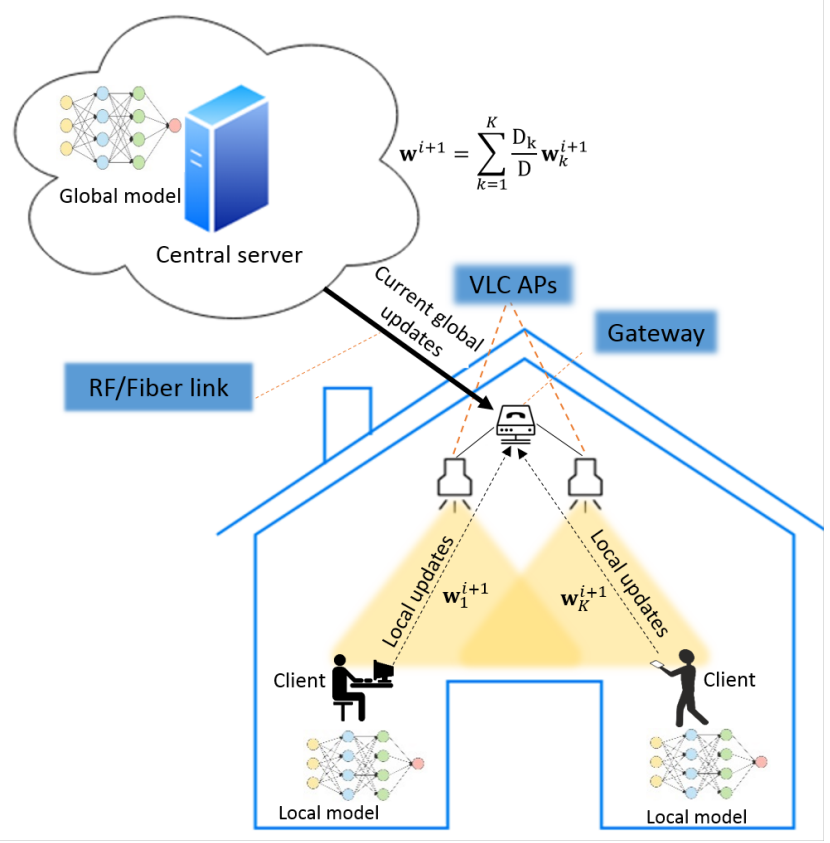

Fig. 2: System architecture of FL in VLC.

the following operation

$$
\mathbf{w}^{i+1}=\mathbf{w}^{i}-\eta \frac{1}{D} \sum_{k=1}^{K} D_{k} \Delta F_{k}\left(\mathbf{w}^{i}\right)
$$

where $D$ is the total data size of participating clients, $\eta$ is the learning rate, and $\Delta$ is the derivative operation. Thus, the server updates the global model parameters based on the weighted average of the attained local parameters. Following this, the server shares the updated global model parameters with the clients in the next iteration to enhance the accuracy of the global model. Notably, weights exchange is performed over multiple rounds until a certain model accuracy level is satisfied. Also, there are several variants of aggregation models that have been proposed to enhance the performance of FedAvg scheme, such as FedProx, FedPAQ, Turbo-Aggregate, FedMA, and HierFAVG.

\section{Design Aspects of FL IN VLC}

\section{A. Client Selection and Scheduling}

Client selection and scheduling constitute an important factor in the implementation of FL in VLC because of its effect on the accuracy and convergence time of the training process. In this regard, the need for developing efficient client selection and scheduling schemes stems from the heterogeneity of clients datasets, devices diverse computational capabilities, available resources, and wireless channel conditions.

Thus, a random selection of clients to participate in the FL process could reduce its efficiency. For example, selecting a client with limited computational capabilities or severe channel conditions will require additional time to compute its updated local model parameters and send them to the server, whilst it will drain the client resources. Accordingly, this will result in a delayed global model aggregation procedure, that is needed to accomplish the scheduled training process. Hence, a key factor to improve the training convergence time and achieve a high-performance training relies on how to properly select the participating clients and assign the training tasks among them.

Efficient clients selection and scheduling should be performed while considering numerous aspects. Typical VLC indoor environment is usually deployed with multiple LEDs, each with limited coverage area. Therefore, in order to provide ubiquitous coverage each LED acts as a VLC access point (AP) that handles multiple clients located in its coverage area. Consequently, clients and AP association is the first step towards realizing efficient client selection and scheduling, and subsequently successful implementation of FL in indoor VLC environments. It is also recalled that in order to achieve acceptable performance in FL, a considerable number of clients should participate in the local training process. Consequently, this will lead to an increased communication overhead, due to the limited bandwidth of the uplink and downlink channels, as well as the constrained energy resources. Hence, developing effective resource management schemes for the uplink and downlink is essential in minimizing resources consumption, while maximizing global model accuracy. Most of the available resource management techniques rely on formulating optimization problems that are handled by heuristic or reinforcement learning tools. As most of these techniques are developed for RF communications, their extension to VLC systems needs be revisited.

From a different angle, a larger number of clients does not readily imply faster model convergence, which is due to the increased heterogeneity of the data among clients and the waiting time, which may cause additional delay. Moreover, reliability of the participating clients is another issue related to increasing the number of the clients. Hence, the optimum number of participating clients should be maximized while taking into consideration these limitations. Within the same context, straggler clients, which are dropped from the learning process due to their low battery levels, the absence of LoS links, or connectivity issues, is a common problem in FL that yields a wasted resources of both the server and other clients. Several research contributions shed light on these issues, proposing numerous techniques to overcome straggler clients problem, including, redundancy and asynchronicity. Finally, given that global parameters in the downlink are carried over the light intensity, maintaining the communication while lights are off by switching to RF could guarantee successful implementation of FL in VLC. Specifically, a switch to conventional RF clients scheduling schemes should be implemented in the off-light mode. 


\section{B. Joint Communication and Learning}

It is recalled that the communication process in FL for VLC is carried out over two different wireless media. Specifically, the first one is the downlink communication which is realized through optical signals for sharing the global model parameters after aggregating them at the server. The uplink is realized over RF or infrared signals for uploading the updated local models to the server. Indeed, communication over wireless media is usually unreliable due to the effect of different impairments such as noise, shadowing, fading, and path loss. In addition to that, VLC introduces additional impairments, including, ambient light and random receiver orientation. Hence, the accuracy of the model and the convergence time of FL is highly dependent upon the channel impairments, that may introduce significant training errors.

In light of this, in order to ensure a realistic and accurate implementation of FL in VLC, the effect of the transmission errors in the uplink and the downlink need be addressed. To this end, different error detection codes such as, parity checking, cyclic redundancy check, or longitudinal redundancy check can be utilized, aiming to determine if the global and local models are erroneously received. Subsequently, the server will discard the invalid local model updates, and aggregate the error-free local model updates only. Similarly, clients who experience a degraded optical signal in the downlink global model transmission may be discarded from the training process in a particular iteration. Moreover, by leveraging error correction codes at the LEDs side, the clients will be able to detect a certain number of errors in the corrupted global model, and then correct them to avoid global model re-transmission.

\section{Model Compression}

It is recalled that in large-scale FL-enabled networks, a large number of parameters updates needs be exchanged in each communication round. Hence, research efforts have been devoted in achieving communication-efficient implementation of FL. Specifically, three main directions exist, namely, model updates size reduction, communication frequency reduction, and communication type.

The aim of model updates size reduction is to reduce the size of the messages carrying model updates, in both uplink and downlink, through lossy and lossless compression techniques. To this end, quantization and sparsification (lossy compression techniques) have been extensively investigated in the context of FL. In fact, quantization process compresses each entry of model updates into a single quantum value with finite number of bits, thus reducing its size. One the contrary, in a sparsification procedure, a subset of model updates is selected to be communicated, based on a pre-determined threshold of the model gradient size. Hence, gradients with sizes smaller than the threshold value will be replaced by zeros while the larger ones are only transmitted.

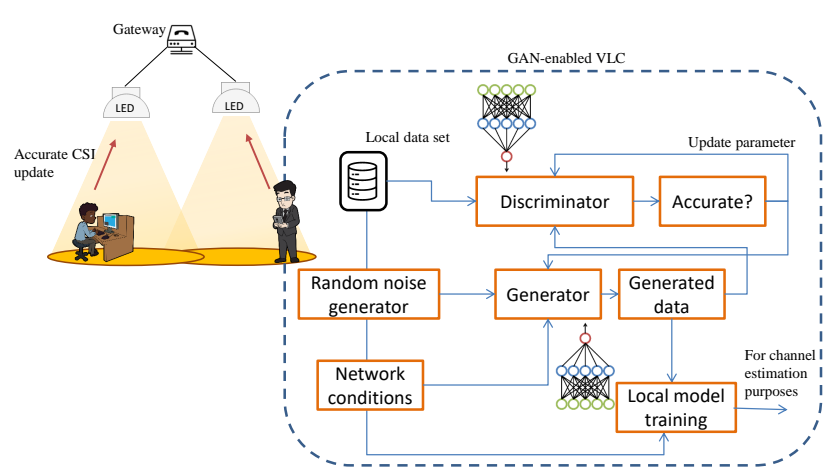

Fig. 3: GAN-enabled VLC system for enhanced CSI acquisition through FL.

\section{Users Mobility Behaviour Prediction}

Modeling and predicting users' mobility in indoor VLC environment plays an important role in analyzing different communication design aspects. In particular, mobility prediction constitutes an efficient tool for location update, radio resource management, signaling traffic needed for handover, and users' association. In FL, users mobility limits the performance of FL in VLC networks. This stems from the fact that the nature and amount of available training datasets vary with mobility, in addition to channel state information (CSI) fluctuation. Therefore, enhancing model training and aggregation accuracy of local updates in FL, requires consideration of users' mobility.

Two different approaches are presented in the literature for individual mobility prediction, namely, personal mobility model with local-information and joint mobility model with population information. In the former, user's local mobility data is utilized to predict its own mobility behaviour. In such techniques, overcoming the sparsity of the mobility data records requires collaborative model training. To that end, FL can be leveraged, through a large number of clients, to evaluate a global generalized model that can be utilized for mobility prediction. Also, by leveraging mobility prediction models, clients selection can be preformed according to the mobility behavior of each user. Hence, only users with low mobility can participate in local model training, in order to prevent transmission errors that may occur during local model updates.

\section{OpEn RESEARCH DIRECTIONS}

\section{A. Generative Adversarial Networks for Enhanced VLC Channel Estimation}

FL was recently considered in distributed CSI acquisition. Also, it was effective in data transmission overhead reduction compared to centralized learning, while ensuring reliable model training and acceptable level of channel estimation accuracy [13]. In this context, local datasets at each participating device may fail to capture the VLC channels behavior in different scenarios, including the presence of ambient 
light noise, receiver random orientation, shadowing, and user mobility. In particular, when VLC channel conditions vary due to specific scenarios, it is essential to re-estimate the channel using pilots, and then collect data and update the local models accordingly. This will result in an increased pilot overhead, and therefore a higher loss in energy and time. In light of this, developing generalized models for accurate channel estimation in VLC, local models should be trained while considering extreme network cases, imposing additional challenges on the implementation of FL in VLC networks.

To this end, generative adversarial networks (GANs) represent an efficient solution to create a generalized framework that experienced a wide range of special network conditions [14]. Specifically, in a GAN, a generator, which is enabled by a deep neural network, is trained to generate close-to-real channel data, and then a discriminator is utilized to quantify the learning accuracy. By leveraging GANs, the limited local datasets, representing the behavior of VLC channels under particular scenarios, will be extended to comprise real and synthetic data, covering all network conditions. Therefore, improved training models can be accomplished, and hence a more accurate and generalized channel estimation can be acquired.

From a different angle, GAN algorithm requires a large dataset for accurate samples generation. Therefore, the learning process, synthetic data generation accuracy, and hence, the reliability of the estimated channel behavior is constrained by the limited available channel samples at each device. In this regard, an extended version of GAN is proposed in [15] to achieve network-wide channel estimation and modeling, in which all participating devices collaborate in order to develop generalized and comprehensive models. This developed GAN architecture is known as distributed GAN (DGAN). Unlike conventional distributed cooperative learning methods, where devices share their datasets for exhaustive training, DGAN brings up the advantage of ensuring users data privacy. In particular, participating devices in DGAN share locally generated synthetic data, instead of their private raw data.

As a promising algorithm, research efforts should be directed towards implementing GANs in VLC systems, outlining implementation challenges, practical design aspects, and highlighting possible applications.

\section{B. Reconfigurable Intelligence Surfaces}

The reconfigurable intelligent surface (RIS) concept was recently identified as a key enabler for beyond 5G networks, offering extended coverage, enhanced signals reliability, and improved energy efficiency. RIS comprises a number of reconfigurable metasurfaces with unique artificially-manipulated electromagnetic properties, enabling them to control/adjust the properties of impinging wireless signals. This can be achieved by enabling a wide range of functionalities, including beam focusing, splitting, reflection, absorption, and polarization. Therefore, RIS can be of particular interest in FL-enabled
VLC systems from two different perspectives, namely, RISassisted and RIS-equipped LEDs. Regarding the former, multiple RISs can be mounted on the walls of an indoor area to enable a number of functionalities that assist with global models transmission. Specifically, RIS can play a vital role in assisting the establishment of LoS links between participating devices and the server. It is recalled that having a LoS link is an essential component in VLC systems, and therefore, any blockage yields a service interruption. In this context, RIS can be a promising candidate to tackle this issue [Fig. 4, (1)]. Also, signals reflected from the RIS can be used for energy harvesting purposes, allowing power-constrained device to communicate their models reliably [Fig. 4, (2)].

On the contrary, with a proper tuning of an RIS, which is placed at the transmitter front-end, beam focusing can be realized by a controlled adjustment of the LED's FoV. This results in an improved global model reception and increased number of participating devices attributed to the improved coverage [Fig. 4, (3)]. Within the same context, an RIS can be exploited to enhance the physical layer security, by blocking the transmitted global models and prevent them from potential eavesdroppers [Fig. 4, (4)]. However, such promising advantages, attained by the integration of RIS in FL-enabled VLC systems, can be realized only if the RIS parameters are properly optimized and tuned to deliver the anticipated outcomes. It is worth highlighting that the optimization of FLenabled VLC with RIS has not been touched in the literature yet, rendering it an attractive open research problem.

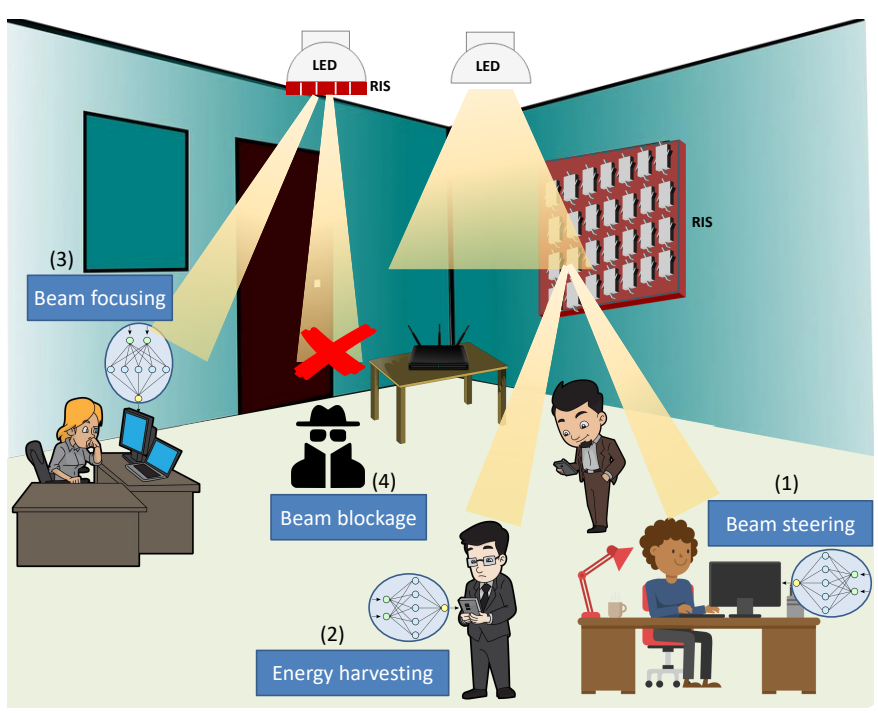

Fig. 4: RIS-enabled FL for VLC scenario.

\section{Multiple Access}

Multiple access (MA) schemes are indispensable parts of future network generations, fulfilling the massive scale connectivity associated with emerging applications. In VLC, several optical orthogonal multiple access schemes exist, 
including time-division multiple access, orthogonal frequencydivision multiple access, and optical code-division multiple access. On the contrary, space-division multiple access exploits the spatial separation between users to provide full time and frequency resources. Furthermore, non-orthogonal multiple access has been recognized as a spectrally-efficient multiple access scheme that allows different users' signals to be multiplexed in power domain, sharing the same frequency resources simultaneously.

Notably, the aforementioned schemes fundamentally rely on advanced optimization algorithms, in order to coordinate users access to the network resources. Owing to the inherent nonconvexity and infinite dimensionality of these optimization problems, iterative algorithms are usually exploited with the aim to obtain the optimum resource allocation scheme, allowing fair users access to the network. Despite the satisfying performance achieved by different optimization tools in MA schemes, their performance is generally constrained by the high computational overhead, which hinders their real-time implementation. Moreover, due to the dynamic nature of VLC networks, a frequent execution of the iterative algorithms will occur.

Conventionally, classical ML constitutes the optimum tool to facilitate solving such optimization problems, with the aid of sensory data transmitted from the clients, such as current allocated spectrum, device non-linearity information, and the presence of interfering signals. However, ML algorithms have shown some shortage in terms of privacy, delay, and energy consumption, and hence, FL can be a prominent alternative to generate locally trained models. In this regard, the global feedback mechanism in FL allows participating devices to utilize the globally trained model to perform on-site resource allocation optimization, and hence, achieve cooperative coordinated network access.

\section{FL in Hybrid RF/VLC Systems}

Typically, in VLC, light emitted from LEDs is confined within small areas, limiting the participating devices to the ones exist in the LEDs coverage area. Since VLC can provide interference-free communication, with co-existing $\mathrm{RF}$ systems, hybrid integration of RF and VLC is expected to provide ubiquitous coverage and enhanced user experience. In a hybrid RF/VLC architecture, each LED serves as an AP to provide high data rate transmission, and is supported by one or multiple RF APs that guarantee uninterrupted moderate data rate transmission, in case of blockage. Hence, each user within the indoor environment is associated with either a VLC or RF AP.

It is recalled that user selection is one of the most challenging issues in FL, particularly in RF systems due to the limited resources. Therefore, hybrid RF/VLC architecture constitutes an appealing solution to enhance the performance of FL by: i) allowing a larger number of users to participate in the model training process; ii) establish communication links for VLC clients in case of blockage. However, to ensure efficient integration of FL in hybrid RF/VLC systems, its performance needs be optimized by considering APs-users association and resource allocation.

\section{E. FL for Augmented Reality Applications in VLC}

Augmented reality (AR) application is one of the latest technology trends, emerged to provide interactive and immersive users experience, by combining virtual visual and auditory contents with real environments. AR spans a variety of applications in different disciplines starting from TV and films production, weather sciences, disaster relief, medicine, education, and entertainments. To provide immersive experience over the real world, these AR devices are equipped with cameras, GPS modules, and sensors. However, AR applications are highly localized and sensitive to latency issues. Meanwhile, AR applications generate enormous data from multiple users such as images, which requires an intensive data processing capabilities and bandwidth resources. Typical high quality AR applications requires data rates of multiple Gbps. Moreover, with the accelerating demands for multiobject virtualization, the accuracy of detection and classification is essential to enhance users immersive experience. Hence, overcoming latency and enhancing clients privacy whilst reducing communication overhead, demanding AR algorithms can be processed at the AR users side with the aid of FL. Conversely, VLC is characterized by the ability to provide secure high data rate communication. Therefore, it can establish high speed wireless links between a centralized server and AR clients to offload models update traffic from the current crowded RF spectrum, as a way to overcome the aforementioned limitations. Hence, integrating FL into VLC constitutes an important part in enhancing users experience in AR applications.

\section{CONCLUSION}

We addressed the potential integration of the newly emerged FL paradigm in VLC systems. In particular, we presented a brief background about VLC technology and the basic concepts of FL and aggregation mechanisms. Subsequently, we provided the fundamentals for integrating FL into VLC systems and highlighted design aspects and promising solutions. Finally, we outlined some envisioned future research directions, which need be investigated prior to real implementation of FL VLC systems.

\section{REFERENCES}

[1] F. Musumeci, C. Rottondi, A. Nag, I. Macaluso, D. Zibar, M. Ruffini, and M. Tornatore, "An overview on application of machine learning techniques in optical networks," IEEE Commun. Surveys \& Tuts., vol. 21 , no. 2, pp. 1383-1408, 2018.

[2] H. Huang, S. Guo, G. Gui, Z. Yang, J. Zhang, H. Sari, and F. Adachi, "Deep learning for physical-layer 5G wireless techniques: Opportunities, challenges and solutions," IEEE Wireless Commun., vol. 27, no. 1, pp. 214-222, 2020. 
[3] O. A. Wahab, A. Mourad, H. Otrok, and T. Taleb, "Federated machine learning: Survey, multi-level classification, desirable criteria and future directions in communication and networking systems," IEEE Commun. Surveys Tuts., pp. 1-1, 2021.

[4] P. Kairouz, H. B. McMahan, B. Avent, A. Bellet, M. Bennis, A. N. Bhagoji, K. Bonawitz, Z. Charles, G. Cormode, and R. Cummings, "Advances and open problems in federated learning," arXiv preprint arXiv:1912.04977, 2021.

[5] Q. Li, Z. Wen, Z. Wu, S. Hu, N. Wang, Y. Li, X. Liu, and B. He, "A survey on federated learning systems: Vision, hype and reality for data privacy and protection," 2021.

[6] W. Y. B. Lim, N. C. Luong, D. T. Hoang, Y. Jiao, Y. C. Liang, Q. Yang, D. Niyato, and C. Miao, "Federated learning in mobile edge networks: A comprehensive survey," IEEE Commun. Surveys Tuts., vol. 22, no. 3, pp. 2031-2063, 2020.

[7] T. Li, A. K. Sahu, A. Talwalkar, and V. Smith, "Federated learning: Challenges, methods, and future directions," IEEE Signal Process. Mag., vol. 37, no. 3, p. 50-60, May 2020. [Online]. Available: http://dx.doi.org/10.1109/MSP.2020.2975749

[8] S. Niknam, H. S. Dhillon, and J. H. Reed, "Federated learning for wireless communications: Motivation, opportunities and challenges," arXiv preprint arXiv:1908.06847, 2020.
[9] M. Aledhari, R. Razzak, R. M. Parizi, and F. Saeed, "Federated learning: A survey on enabling technologies, protocols, and applications," IEEE Access, vol. 8, pp. 140 699-140725, 2020.

[10] L. Lyu, H. Yu, and Q. Yang, "Threats to federated learning: A survey," arXiv preprint arXiv:2003.02133, 2020.

[11] J. Posner, L. Tseng, M. Aloqaily, and Y. Jararweh, "Federated learning in vehicular networks: Opportunities and solutions," IEEE Netw., vol. 35, no. 2, pp. 152-159, 2021.

[12] Q. Yang, Y. Liu, T. Chen, and Y. Tong, "Federated machine learning. Concept and applications," arXiv preprint arXiv:1902.04885, 2019.

[13] A. M. Elbir and S. Coleri, "Federated learning for channel estimation in conventional and IRS-assisted massive MIMO," arXiv preprint arXiv:2008.10846, 2020.

[14] A. T. Z. Kasgari, W. Saad, M. Mozaffari, and H. V. Poor, "Experienced deep reinforcement learning with generative adversarial networks (GANs) for model-free ultra reliable low latency communication," IEEE Trans. Commun., Feb. 2020.

[15] Q. Zhang, A. Ferdowsi, W. Saad, and M. Bennis, "Distributed conditional generative adversarial networks (GANs) for data-driven millimeter wave communications in UAV networks," arXiv preprint arXiv:2102.01751, 2021 